\title{
O papel do cirurgião dentista na equipe multiprofissional da Atenção Básica em Saúde, após 19 anos de sua implantação
}

The role of the dentist in the multidisciplinary team of Primary Health Care, 19 years after its implementation El papel del odontólogo en el equipo multidisciplinar de Atención Primaria a la Salud, 19 años después de su implantación Artur Antônio Guedes GURGEL FILHO ${ }^{1}$ Regiane Cristina do AMARAL ${ }^{2}$

${ }^{1}$ Residência Multiprofissional em Saúde da Família e Comunidade na Escola de Saúde Pública do Ceará ${ }^{2}$ Professora Adjunta do Curso de Odontologia da Universidade Federal de Sergipe - Aracaju -SE, Brasil

\section{Resumo}

A Odontologia no Brasil, por muitos anos, passou por modelos assistenciais (Incremental e Simplificado). Houve predomínio do desempenho em um modelo técnico e curativo, direcionado a grupos como escolares. Com o advento do SUS (Sistema Único de Saúde) e posterior criação do Programa Saúde da Família (PSF) em 1994, houve inserção da odontologia na Estratégia Saúde da Família (ESF) no ano 2000. Objetivo: O presente estudo teve como objetivo analisar a percepção dos profissionais que trabalham na ESF com a participação do cirurgião-dentista (CD) na ESF e da equipe do NASF (Centro de Apoio à Saúde da Família) após 19 anos de integração com o SUS. Metodologia: Este estudo transversal foi realizado através de questionários aplicados ao CD, membros da equipe de referência (ESF) e apoio (NASF) da Atenção Básica. Os questionários foram compostos por 11 perguntas, diferentes para as categorias profissionais, sendo auto administrados. Resultados: foram entrevistados 89 profissionais pertencentes às Unidades de Saúde de 15 CRES (Saúde Regional), 69 do sexo feminino e 20 do masculino, 58,4\% com idade entre 20 e 36 anos e $27 \%$ com idade entre 36 e 50 anos. Dos profissionais entrevistados, $94 \%$ consideraram relevante a interação do cirurgião-dentista com a ESF e 80\% perceberam a atitude do CD ou algum aspecto da liderança em ações coletivas. Conclusão: Após 19 anos de integração da saúde bucal, equipes na atenção básica do SUS, observou-se a incorporação do dentista com aspectos positivos.

Descritores: Sistema Único de Saúde; Saúde Pública; Estratégia de Saúde da Família; Odontologia.

\section{Abstract}

Dentistry in Brazil for many years gone through assistance models (Incremental and Simplified). There was a predominance of performance in a technical and curative model, aimed in groups such as schoolchildren. With the advent of SUS (Unified Health System) and subsequent creation of the Family Health Program (PSF) in 1994, there was insertion of dentistry in the Family Health Strategy (ESF) in the year 2000. Objective: The present study aimed to analyze the perception of professionals working in the ESF with the participation of the Dental Surgeon (CD) in the ESF and the NASF team (Family Health Support Center) after 19 years of their integration with SUS. Methodology: This cross-sectional study was carried out through questionnaires applied to the DC, members of the reference team (ESF) and support (NASF) of Primary Care. The questionnaires were composed of 11 questions, different for professional categories, being self-administered. Results: 89 professionals belonging to the Health Units of 15 CRES (Regional Health) were interviewed, 69 of whom were female and 20 male, $58.4 \%$ aged between 20 and 36 years and $27 \%$ aged between 36 and 50 years. Of the professionals interviewed, $94 \%$ considered the interaction of the dental surgeon with the FHS to be relevant and $80 \%$ perceived the attitude taken by the DC or some aspect of leadership with collective actions. Conclusion: After 19 years of integration of the oral health, teams in the primary care of SUS, the incorporation of the dentist with positive aspects were observed.

Descriptors: Unified Health System; Public Health; Family Health Strategy; Dentistry.

\section{Resumen}

La Odontología en Brasil durante muchos años pasó por modelos de asistencia (incremental y simplificado). Predominó el desempeño en un modelo técnico y curativo, dirigido a grupos como escolares. Con el advenimiento del SUS (Sistema Unificado de Salud) y la posterior creación del Programa de Salud Familiar (PSF) en 1994, se insertó la odontología en la Estrategia de Salud Familiar (ESF) en el año 2000. Objetivo: El presente estudio tuvo como objetivo analizar La percepción de los profesionales que trabajan en el FSE con la participación del cirujano dental (CD) en el FSE y el equipo NASF (Centro de Apoyo a la Salud Familiar) después de 19 años de su integración con el SUS. Metodología: este estudio transversal se realizó a través de cuestionarios aplicados a DC, miembros del equipo de referencia (ESF) y apoyo (NASF) de Atención Primaria. Los cuestionarios estaban compuestos por 11 preguntas, diferentes para categorías profesionales, autoadministradas. Resultados: se entrevistó a 89 profesionales pertenecientes a las Unidades de Salud de 15 CRES (Salud Regional), 69 de los cuales eran mujeres y 20 hombres, el 58,4\% tenían entre 20 y 36 años y el $27 \%$ tenían entre 36 y 50 años. De los profesionales entrevistados, el 94\% consideró que la interacción del cirujano dental con el FHS era relevante y el $80 \%$ percibió la actitud adoptada por el DC o algún aspecto del liderazgo con acciones colectivas. Conclusión: Luego de 19 años de integración de la salud bucal, se observaron equipos en la atención primaria del SUS, la incorporación del dentista con aspectos positivos.

Descriptores: Sistema de Salud Unificado; Salud Pública; Estrategia de Salud Familiar; Odontología.

INTRODUÇÃO

O Programa Saúde da Família (PSF), atualmente ESF (Estratégia de Saúde da Família) surge no Brasil como uma estratégia de organização do modelo assistencial a partir da atenção básica, em conformidade com os princípios e diretrizes do Sistema Único de Saúde (SUS). Seu objetivo de reordenar e dar um novo acesso as práticas de saúde no âmbito da atenção básica embasados nos critérios, com foco na família, a partir do seu ambiente físico e social ${ }^{1,2}$.

Sendo assim, o PSF surge como um novo jeito de trabalhar a saúde, tendo a família como centro de atenção e não somente o indivíduo doente, implementando uma nova visão no processo de intervenção em saúde na medida em que não espera a população chegar para ser atendida, pois age preventivamente sobre ela a partir de um novo modelo de atenção ${ }^{3}$.

A inserção da odontologia na ESF só aconteceu no ano de 2000, quando o Ministro da Saúde, diante da necessidade de ampliar a atenção em saúde bucal para a população brasileira, estabeleceu incentivo financeiro para a formação de equipes formadas por cirurgiões- 
dentistas (CDs), auxiliares em saúde bucal (ASB) e técnicos em saúde bucal (TSB) ${ }^{4}$.

A Odontologia no Brasil por muitos anos passou por modelos assistenciais (Incremental e Simplificado). Houve um predomínio de atuação basicamente num modelo técnico e curativo, voltado em partes a pequenos grupos sociais como os escolares ${ }^{5,6}$. Assim, diante da criação da ESF foi necessário a readequação deste profissional a fim de que a odontologia se articulasse com outros setores da saúde para que pudesse consolidar a construção de um novo conceito de saúde mais eficiente, agregador, humano e integral e de modo holístico ${ }^{7}$.

Assim, o presente estudo teve como objetivo analisar e suscitar o dia a dia da ESF com a participação do CD, após 19 anos de sua integração no SUS, bem como suas relações de trabalho, inovação, desenvoltura e as dificuldades encontradas com outros profissionais de saúde, sob a ótica da equipe e do CD.

\section{MATERIAL E MÉTODO}

Trata-se de um estudo transversal que foi realizado por meio de questionários aplicado ao $C D$, membros da equipe de referência (ESF) e apoio (NASF) da Atenção Básica. Os questionários foram compostos por 11 perguntas, diferentes para categorias profissionais, sendo autoaplicado.

Participaram do estudo gerentes de unidades, médicos, enfermeiros, técnicos de enfermagem, técnicos em saúde bucal, agentes comunitários de saúde, dentistas, assistente social, profissional de educação física, farmacêutico, fisioterapeuta, fonoaudiólogo, profissional com formação em arte e educação (arte educador), nutricionista, psicólogo, fisioterapeutas e terapeuta ocupacional. Estes foram questionados sobre o processo de inclusão do $C D$ junto a equipe multidisciplinar. Para o CD foi questionado sobre as principais dificuldades e desafios encontrados na inclusão.

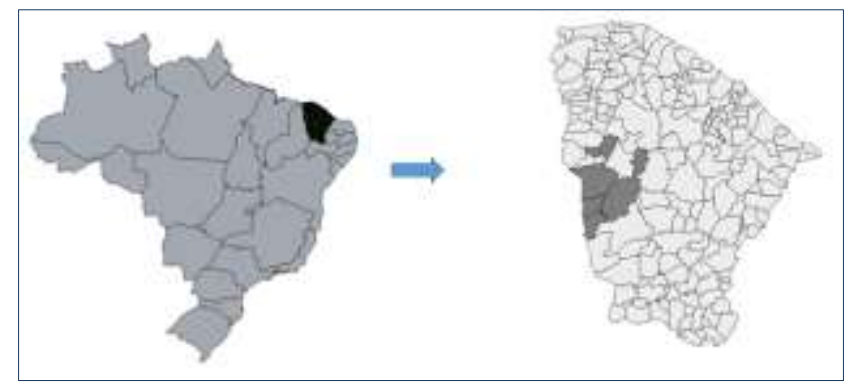

Figura 1: Disposição dos municípios analisados no Mapa.

O estudo foi desenvolvido em Unidades Básicas de Saúde, pertencentes a Regional 15 de Crateús no interior do Ceará (CRES), que tinham entre seu quadro profissional $O C D$.
Entre os municípios pertencentes a esta regional tem-se: Crateús, Novo Oriente, Independência, Nova Russas, Monsenhor Tabosa, Quiterianópoles e Ararendá (Figura 1, Tabelas 1 e 2)

Tabela 1. Número de Equipes de Saúde da Família junto a saúde bucal que compõem os municípios de toda regional de saúde.

\begin{tabular}{|c|c|c|}
\hline Municípios & ESF & Saúde Bucal \\
\hline Ararendá & 5 & 5 \\
\hline Crateús & 22 & 17 \\
\hline Independência & 10 & 10 \\
\hline Ipaporanga & 6 & 3 \\
\hline Ipueiras & 14 & 11 \\
\hline Monsenhor Tabosa & 7 & 4 \\
\hline Nova Russas & 11 & 11 \\
\hline Novo Oriente & 11 & 3 \\
\hline Poranga & 5 & 5 \\
\hline Quiterianópoles & 9 & 4 \\
\hline Tamboril & 12 & 11 \\
\hline
\end{tabular}

Tabela 2. Número de núcleos de Apoio a Saúde da família junto a Estratégia de Saúde da Família nos municípios de toda regional de saúde

\begin{tabular}{|c|c|c|c|c|c|c|c|c|}
\hline \multicolumn{9}{|c|}{ Cobertura de Núcleo de Apoio a Saúde da Família em 2018} \\
\hline \multirow{2}{*}{ Municípios } & \multicolumn{4}{|c|}{ NASF Tipo I } & \multicolumn{4}{|c|}{ NASF Tipo II } \\
\hline & CRMS & CDS & IMPL & CB & CDMS & CDS & IMPL & \% CB \\
\hline Ararendá & 0 & 0 & 0 & 0 & 1 & 1 & 1 & 100 \\
\hline Crateús & 2 & 2 & 2 & 100 & 0 & o & 0 & 0 \\
\hline Independência & 1 & 1 & 1 & 100 & 0 & 0 & 0 & 0 \\
\hline Ipaporanga & 0 & $\mathrm{o}$ & 0 & 0 & 0 & 0 & 0 & o \\
\hline Ipueiras & 1 & 1 & 1 & 90 & o & 0 & 0 & o \\
\hline $\begin{array}{l}\text { Monsenhor } \\
\text { Tabosa }\end{array}$ & 0 & 0 & 0 & 0 & 1 & 1 & 1 & 66,6 \\
\hline Nova Russas & o & 1 & o & 0 & 1 & 0 & 0 & 0 \\
\hline Novo Oriente & 1 & 1 & 1 & 81,8 & 0 & 0 & 0 & 0 \\
\hline Poranga & 0 & 0 & 0 & 0 & 1 & 1 & 1 & 100 \\
\hline Quiterianópoles & 0 & o & 0 & 0 & 1 & 1 & 1 & 44,4 \\
\hline Tamboril & 1 & 1 & 1 & 81,8 & 0 & 0 & o & 0 \\
\hline
\end{tabular}

Após consulta à escala dos profissionais foi elaborado um cronograma de visitas planejando encontrar o máximo de profissionais nos dias visitados. Todos os profissionais que trabalham das UBS e equipe NASF foram convidados por escrito mediante carta de apresentação e pessoalmente para fazer parte da pesquisa. Os que desejaram participar assinaram o TCLE (Termo de Consentimento Livre e Esclarecido) do Comitê de ética em pesquisa (06971019.0.1001.5546). As análises dos dados foram de forma descritiva, utilizando o programa Microsoft Excel.

RESULTADOS

Foram entrevistados 89 profissionais pertencentes às Unidades de Saúde da 15 CRES, sendo 69 gênero feminino e 20 masculino, $58.4 \%$ com idade entre 20 a 36 anos e $27 \%$ entre 36 e 50 anos.

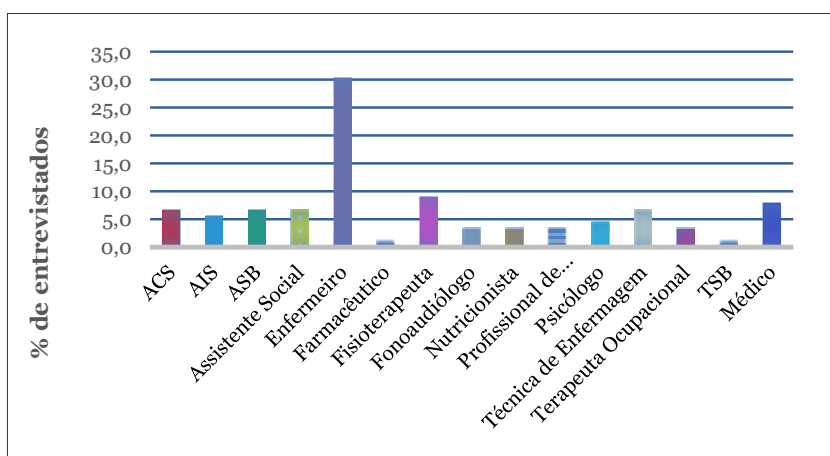

Figura 2. Percentual de profissionais entrevistados. 15 CRES, 2019. 
Tabela 3. Questionamentos aos profissionais de saúde sobre a participação e integração do profissional cirurgião dentista frente as atividades na Unidade de Saúde. 15 CRES, 2019

Você julga relevante a integração e participação do cirurgião dentista na Equipe de Saúde da Família?

Não

Sim

Como é o seu convívio profissional, diário com o cirurgião dentista?

Bom

Ruim

O dentista participa junto com a Equipe de Saúde da Família dos momentos de

discussão e programação e estratégias desenvolvidas?

Sim, sempre.

Sim, às veze

Nunca.

Caso Sim, Como se dá a relação e participação do dentista a frente destas reuniões?

Muito Participativo

Participativo

Fala apenas quando solicitado

Não participativo

Você vê no dentista da sua Unidade um potencial idealizador de projetos?

Sim

Não se

Não

Como se dá suas ações coletivas na qual há a participação do dentista?

Bom

Muito Bom

Ruim

Nunca acontecen

Você percebe a tomada de atitudes ou algum aspecto de liderança do cirurgião

dentista durante as atividades de ações coletivas em saúde?

Sim, com muita frequência.

Sim, porém com pouca frequência.

Não.

Você vê no Dentista da sua unidade como um potencial criador e inovador em ações coletivas?

coletiva

Não se

Não.

60

Tabela 4. Questionamentos Cirurgiões dentistas sobre a integração com a equipe multidisciplinar nas atividades na Unidade de Saúde. 15 CRES, 2019

Quanto tempo de carreira como cirurgião-dentista?

Abaixo de 5 anos

Entre 5 e 10 anos

Entre 10 e 20 anos

\begin{tabular}{|l|l|}
\hline Como você considera a sua vivênci em aúde pública, durante a sua graduã̃o? & 2
\end{tabular}

Como vaúde pública, durante a sua graduação?

\begin{tabular}{l} 
Excelente \\
\hline Bom, porém não foi possível contemplar tudo que o SUS exigia \\
\hline
\end{tabular}

Ruim, me forneceu porco ou neque sus exigia.

Ruim,

Durante a graduação você foi preparado para realizar trabalhos em equipe?

Sim

Não

Como você analisa e compara a capacidade de criar ou inovar frente às adversidades

da Saúde Pública com o que lhe foi preparado pela graduação em odontologia?

Entendo que todos nós podemos desenvolver tais habilidades de acordo

com o auxílio de que a graduação possa nos dar para desenvolver essas

habilidades

Entendo que essas habilidades são inatas e individuais de cada

profissional, independente da graduaç̃o.

Entendo que mesmo a raduac̃̃o ajudando a desenvolver tais

habilidades anuns profissionais pode negligenciar

hurante a graduacão você acredita que foi preparado para desen

para criar ou inovar frente às adversidades da saúde pública?

para cira

Sim, porém faltou um pouco mais de preparo.

Não.

\begin{tabular}{l|l}
\hline & 7 \\
\hline & 5
\end{tabular}

saúde coletiva ou saúde da família?

Não

Sim

Se sim, acredita que ele te preparou para trabalhar de acordo princípios e diretrizes do SUS?

Não

Sim

Se sim acredita que o seu curso de pós-graduação te preparou para o trabalho em equipe?

Sim.

Sim.

Sim, por

Se sim durante a pós-graduação acredita que foi preparado para desenvolver seu

potencial para criar ou inovar frente às adversidades da saúde pública?

Sim.

Sim, porém os anos e a experiência profissional complementaram essa

habilidades e conhecimento.

Não.

DISCUSSÃO

Com a implantação do Sistema Único de Saúde no Brasil, inúmeras transformações foram constatadas no campo da saúde, baseadas nos novos conceitos em relação aos saberes e práticas que acreditavam na atuação diferenciada dos profissionais frente ao processo saúde/doença, de forma holística e integral dentro da Saúde ${ }^{8}$. Assim, atualmente dentro da ESF tenta-se junto a equipe e nas equipes auxiliares como o NASF, interação para que 0 paciente tenha suas necessidades atendidas e tenha resolutividade em sua atenção.

Através dos dados do presente estudo a maioria dos entrevistados cirurgiões-dentistas tem menos de 5 anos de carreira, ou seja, foram inseridos nas novas diretrizes curriculares nacionais direcionadas pelo Ministério da Educação, sobre a importância do mesmo em atividades junto ao SUS (Sistema Único de Saúde). Reflexo disso são as respostas das questões sobre que a maioria respondeu que seu curso de graduação o preparou para atividades em equipe. Quando é questionado ao CD sobre sua formação, a maioria considera bom, contudo $58 \%$ considera que havia necessidade de mais estudos para aprender sobre o SUS. Assim a importância cada vez maior do estudante estar inserido dentro dos serviços de saúde. Afinal, atualmente, o SUS é - único sistema de saúde para aproximadamente 140 milhões de brasileiros ${ }^{9,10}$ e ainda constitui-se numa importante opção de mercado de trabalho para os profissionais da Odontologia, principalmente a partir da inserção da saúde bucal na Estratégia do Programa de Saúde da Família ${ }^{10}$.

Contudo, o papel do cirurgião dentista deixou de ser somente curativo após o SUS, o dentista precisou assumir modelos de atenção à saúde. O cirurgião-dentista deve participar de equipes interdisciplinares e multiprofissionais para 0 planejamento de políticas públicas saudáveis e o desenvolvimento de ações de vigilância da saúde da coletividade ${ }^{11}$.

Ao questionar a equipe sobre o cirurgião dentista verifica-se que $94 \%$ julgam relevante a interação do cirurgião dentista junto a ESF e $80 \%$ percebe a tomada de atitudes ou algum aspecto de liderança junto a ações coletivas.

Mattos e al. ${ }^{12}$ encontraram dentistas resistentes ao trabalho em equipe, contudo os autores alegam que isso se deu pelo fato da mudança repentina no modo de trabalho da equipe de saúde bucal, que os mesmos não receberam treinamento para o mesmo. Sendo sugerido uma capacitação profissional de saúde frente as mudanças, principalmente junto ao Programa Brasil Sorridente ${ }^{13,14}$. O Estado do Ceará foi um dos pioneiros do PAC (Programa das Agentes Comunitárias em Saúde), criado em 1991 na tentativa de diminuir a mortalidade infantil e desnutrição ${ }^{15}$. O PAC foi um programa pioneiro da ESF, assim este trabalho de 
promoção da saúde tenha maior adesão por parte dos profissionais.

No estudo de Lourenço et al. $^{16}$, ao analisar a interação do cirurgião dentista junto a ESF, os cirurgiões relataram que esta interação não se manifesta no planejamento e/ou na troca de saberes, relatando que $48,9 \%$ dos cirurgiões dentistas não receberam capacitação. A integração entre ESB e Equipes de Saúde da Família (ESF) foi verificada em 76,2 \%; todavia, apenas $54 \%$ realizam reuniões frequentes. Diferente dos dados do presente estudo em que 93\% dos entrevistados (profissionais de saúde em geral) declarou que o dentista participa junto com a Equipe de Saúde da Família dos momentos de discussão e programação e estratégias desenvolvidas.

Segundo a PNSB (Política Nacional de Saúde Bucal) ${ }^{13}, 75 \%$ a $85 \%$ da carga horária contratada do $C D$ devem ser dedicadas à assistência clínica ${ }^{17}$. Assim, durante a maior parte do tempo, o trabalho do $C D$ é caracterizado de forma privada, dificultando até mesmo a interação de reuniões dentro da equipe. ${ }^{18}$ Observa-se este fato no presente estudo em que ao se perguntar a participação do CD junto a programação e estratégias desenvolvidas que $54.5 \%$ caracterizam sim, as vezes e $5 \%$ que não.

CONCLUSÃO

As mudanças curriculares e a inserção do cirurgião dentista junto ao SUS auxiliaram na integração do CD junto a equipe. Contudo há necessidade de maior número de equipes de saúde bucal para que o dentista possa ir além de atividades curativas e participar do planejamento da equipe de saúde.

REFERÊNCIAS

1. De Assis F, Mischiati MF. Retrospectiva histórica da implantação do PSF até sua transformação em ESF nos dias de hoje. Uningá Review.2017;3(1):7.

2. Brasil. Ministério da Saúde. Política Nacional de Atenção Básica. Disponível em: http://bvsms.saude.gov.br/bvs/saudelegis/gm/2 017/prt2436_22_09_2017.html

3. Machado CV , Lima LD, Baptista TWF. Políticas de saúde no Brasil em tempos contraditórios: caminhos e tropeços na construção de um sistema universal. Cad. Saúde Pública 2017; 33(Sup 2):e00129616.

4. Viacava F, Oliveira RAD, Carvalho CC, Laguardia J, Bellido JG. SUS: oferta, acesso e utilização de serviços de saúde nos últimos 30 anos. Ciênc saúde coletiva. 2018;23(6): 1751-62.

5. Carnut L, De Araújo Costa SG, De Moura Neto JG. A percepção discente sobre os conteúdos essenciais de Ciências Sociais em Saúde Bucal em uma graduação em odontologia. JMPHC .2017;7(1):39.

6. Narvai PC. Saúde bucal coletiva: caminhos da odontologia sanitária à bucalidade. Rev Saúde Pública. 2006;40(Esp):141-47.

7. Forte FDS, Pessoa TRRF, Freitas CHSM, Pereira CAL, Carvalho Junior PM. Reorientação na formação de cirurgiõesdentistas: o olhar dos preceptores sobre estágios supervisionados no Sistema Único de Saúde (SUS). Interface (Botucatu). 2015; 19(Suppl1):831-43.

8. Souza MC, Araújo TM, Reis Jr WM, Souza JN, Vilela ABA, Franco TB. Integralidade na atenção à saúde: um olhar da Equipe de Saúde da Família sobre a fisioterapia. Mundo Saúde. 2012;36(3):452-60.

9. Brasil. Ministério da Saúde. Fundo Nacional de Saúde. Gestão financeira do Sistema Único de Saúde: manual básico. $3^{\mathrm{a}} \mathrm{ed}$. rev e ampl. Brasília: Ministério da Saúde; 2003. (Série A. Normas e Manuais Técnicos).

10. Pereira AC, Mialhe FL, Pereira SM, Meneghim MC. O mercado de trabalho odontológico em saúde coletiva: possibilidades e discussões. Arq Odontol. 2010;46(4):232-39.

11. Aerts D, Abegg C, Cesar K. O papel do cirurgiãodentista no Sistema Único de Saúde. Ciênc saúde coletiva. 2004;9:131-38.

12. Mattos GCM, Ferreira E, Leite ICG, Greco RM. A inclusão da equipe de saúde bucal na Estratégia Saúde da Família: entraves, avanços e desafios. Cienc saúde coletiva. 2014;19(2):373-82.

13. Brasil. Ministério da Saúde. Programa Brasil Sorridente. Disponível em: https://aps.saude.gov.br/ape/brasilsorridente

14. Brasil. Ministério da Educação. Diretrizes Curriculares. Disponível em: http://portal.mec.gov.br/mais-educacao/323secretarias-112877938/orgaos-vinculados82187207/12991-diretrizes-curriculares-cursosde-graduacao

15. Brasil. Ministério da Saúde. Programa Agentes Comunitárias. [Citado em 17 fevereiro 2019]. Disponível em:http://bvsms.saude.gov.br/bvs/publicacoes/ pacs01.pdf

16. Lourenco EC, Silva ACB, Meneghin MC, Pereira AC. A inserção de equipes de saúde bucal no Programa Saúde da Família no Estado de Minas Gerais. Ciênc saúde coletiva 2009;14(Suppl.1):1367-377.

17. Ministério da Saúde. Diretrizes da Política Nacional de Saúde Bucal. Secretaria de Atenção à Saúde. Departamento de Atenção Básica. Coordenação Nacional de Saúde Bucal. Brasília: Ministério da Saúde, 2004. 
18. Leme PAT, Bastos RA, Turato ER, Meneghin MC. A clínica do dentista na Estratégia Saúde da Família: entre a inovação e o conservadorismo. Physis. 2019;29(1):e290111.
CONFLITO DE INTERESSES

Os autores declaram não haver conflitos de interesse AUTOR PARA CORRESPONDÊNCIA

Regiane Cristina do Amaral Universidade Federal de Sergipe

Rua Claudio Batista, S/N, Santo Antônio 49060-100, Aracaju - SE, Brasil Tel: (79) 218-1701

E-mail: amaralre@yahoo.com.br

Submetido em 18/01/2021 Aceito em 15/07/2021 